\title{
OCORRÊNCIA DE GIARDIA DUODENALIS EM EQÜINOS DO NOROESTE E DO NORTE DO PARANÁ - BRASIL
}

\author{
LAURO VARGAS \\ LUIZPAULO RIGOLON
}

VARGAS, L., RIGOLON, L.P. Ocorrência de Giardia duodenalis em eqüinos do noroeste e do norte do ParanáBrasil. Semina: Ci. Agr., Londrina, v.19, n.1, p.73-75, mar. 1998.

Resumo: Foram analisadas amostras de fezes de 123 eqüinos do noroeste e do norte do Paraná, onde a ocorrencia de Giardia duodenalis foi estimada em $21,1 \%$. Estudaram-se as seguintes variáveis: idade e sexo. $A$ média dos cistos foi de $9,6 \pm 0,8 \times 6,8 \pm 0,6 \mu \mathrm{m}$. Foi empregada a técnica de centrifugaçâo-flutuação de Faust et al. (1938) com sulfato de zinco (massa especifica $=1.180 \mathrm{~kg} / \mathrm{m}^{3}$ ). O material foi coletado de novembro de 1995 a jultho de 1996.

PALAVRAS-CHAVE: Giardia duodenalis, giardiase, eqüinos, epidemiologia.

\section{INTRODUÇÃO}

Alguns isolados de Giardia duodenalis nāo tem especificidade de hospedeiro e os seres humanos e uma variedade de animais vertebrados compartilham este parasita. Desta forma, a maioria dos autores considera a giardíase como uma zoonose (Acha \& Szyfres, 1986; Healy, 1990; Schantz, 1991).

A prevalència de Giardia e o seu papel na patogenia em grandes animais não estấo bem claros, pois somente nos últimos anos aumentou a pesquisa deste protozoário nos referidos hospedeiros (Kirkpatrick, 1989).

Levantamentos da infecçâo por $G$. duodenalis em animais de produçāo, mostram que a prevalência deste parasita provavelmente seja maior do que em animais de estimaçāo e seres humanos. Isto aumenta o interesse sobre a patogenicidade e o potencial zoonótico da giardiase em animais de produçâo (Xiao, 1994).

A giardiase tem apresentado altas taxas de prevalência em animais domésticos no noroeste do estado do Paraná. A prevalência de $G$. dusodenalis em cães de Maringá foi estimada em $15,6 \%$, e os cistos tinham em média 10,1 $\pm 0,9 \times 7,6 \pm 0,8$ um (Vargas, 1993). A prevalência em gatos de Maringá foi de $6,0 \%$ (Vargas et al., 1995a).

Vargas et al. (1994b) identificaram uma prevalência de $23,0 \%$ em ovinos do município de Cidade Gaúcha, no estado do Paraná. Os cistos de $G$. duodenalis, neste hospedeiro, apresentaram valores médios de 10,2 \pm $0,6 \times 7,6 \pm 0,5 \mu \mathrm{m}$ (Vargas et al., 1994a).

Rigolon \& Vargas (1996b) estimaram uma prevalência de $13,1 \%$ em bovinos de leite no noroeste do Paraná. Os cistos de $G$. duodenalis, neste hospedeiro, mediram em média $10,0 \pm 1,0 \times 7,5 \pm 0,6$ $\mathrm{mm}$ (Rigolon \& Vargas, 1996a).

Vargas et al. (1995/96b) nāo identificaram cistos de $G$. duodenalis em amostras de fezes de caprinos, em Maringá.

A prevalência da giardiase e a sua função na diarréia de eqüinos são pouco conhecidas. Há poucos trabalhos sobre Giardia em eqüinos (Xiäo \& Herd, 1994).
Giardia equi foi descrita pela primeira vez por Fanthan em 1921, em dois eqüinos na África do Sul. Os trofozoitos medem $17-21 \times 9-12 \mu \mathrm{m}$ e os cistos $12-16$ $\times 8-9,5 \mu \mathrm{m}$. G. equi se encontra no grupo $G$. duodenalis (Kulda \& Nohynkova, 1977). Varela \& Salsamendi (1958) identificaram Giardia em um eqüino que apresentava diarréia, no Uruguai. Os cistos mediam 12,0 a $16,0 \times$ 8,0 a $9,5 \mu \mathrm{m}$. Bemrick (1968) observou cistos de Giardja em dois potros sem diarréia, nos Estados Unidos. Os cistos mensurados apresentaram uma média de 12,9 $x 8,7 \mu \mathrm{m}$. Manaham (1970) identificou um eqüino infectado por Giardia em amostras de 32 animais que apresentaram diarréia, na Austrălia. Kirkpatrick \& Skand (1985) observaram a giardíase em um eqüino de quatro anos de idade que apresentava diarréia, nos Estados Unidos. A dimensão média de 20 cistos medidos foi de $12,0 \pm 0,2 \times 8,9 \pm 0,1 \mu \mathrm{m}$. Xião \& Herd (1994) identificar am colhidas aleatoriamente amostras de fezes de 123 eqüinos dos seguintes municípios, do norte e noroeste do estado do Paraná: Arapongas. Cornélio Procópio, Mandaguaçu, Mandaguari, Marialva e Maringá. A ocorrencia foi estimada levando em consideração as seguintes variáveis: idade, e sexo. 0 material foi colhido de novembro de 1995 a julho de 1996.

Os animais foram classificados em dois grupos etários: menor ou igual a um ano e maior de um ano e em machos e fêmeas.

\section{COPROLOGIA}

As amostras de fezes foram colhidas diretamente do reto dos animais, em luvas de plástico descartáveis e foram preservadas em refrigerador $\left(5^{\circ} \mathrm{C}\right)$ durante um período máximo de cinco dias. Foi empregada a técnica de concentração pela centrifugação-flutuação de Faust et al. (1938) com sulfato de zinco (massa específica = $\left.1.180 \mathrm{~kg} / \mathrm{m}^{3}\right)$.

Em cada amostra positiva foram medidos 30 cistos, no seu eixo maior e menor, com micrômetro ocular previamente aferido, com aumento de $400 \mathrm{x}$. 


\section{ANÁLISE ESTATÍSTICA}

Os resultados foram submetidos ao teste de Quiquadrado $(\mathrm{P}<0,05)$. Foi calculada a média dos comprimentos dos eixos maiores e menores dos cistos e seus respectivos desvio padrão e coeficiente de variação.

\section{RESULTADO}

A ocorrência de $G$. duodenalis em eqüinos do noroeste e do norte do estado do Paraná foi estimada em $21,1 \%(26 / 123)$.

A ocorrencia foi diretamente proporcional à idade (Tabela 1). Em eqüinos com idade inferior ou igual a. um ano foi de $18,2 \%(8 / 44)$, e em eqüinos com idade superior a um ano foi de $22,8 \%(18 / 79)$.

Tabela 1. Ocorrencia de Giardia duodenalis em eqüinos do noroeste e norte do estado do Paraná, segundo a idade, no período de novembro de 1995 a julho de 1996.

\begin{tabular}{lcccccc}
\hline \multirow{2}{*}{ Idade (anos) } & \multicolumn{2}{c}{$\mathrm{P}^{\circ}$ ositivo } & \multicolumn{2}{c}{ Negativo } & \multicolumn{2}{c}{ Total } \\
\cline { 2 - 7 } & $\mathrm{N}^{\circ}$ & $(\%)$ & $\mathrm{N}^{\circ}$ & $(\%)$ & $\mathrm{N}^{*}$ & $(\%)$ \\
\hline$\leq 1$ & 08 & $(18,2)$ & 36 & $(81,8)$ & 44 & $(100,0)$ \\
$>1$ & 18 & $22,8)$ & 61 & $(77,2)$ & 79 & $(100,0)$ \\
Total & 26 & $(21,1)$ & 97 & $(78,9)$ & 123 & $(100,0)$
\end{tabular}

Náo houve diferença significativa $(P<0,05)$ entre as idades, em relaçāo ao número de animais positivos.

A ocorrência foi semelhante em fêmeas $(20,5 \%)$ e machos $(22,0 \%)$, conforme a Tabela 2 .

Tabela 2. Ocorrencia de Giardia duodenalis em eqüinos do noroeste e norte do estado do

- Paraná, segundo o sexo, no período de novembro de 1995 a julho de 1996.

\begin{tabular}{lcccccc}
\hline \multirow{2}{*}{ Sexo } & \multicolumn{2}{c}{ Positivo } & \multicolumn{2}{c}{ Negativo } & \multicolumn{2}{c}{ Total } \\
\cline { 2 - 7 } & $\mathrm{N}^{\circ}$ & $(\%)$ & $\mathrm{N}^{\prime \prime}$ & $(\%)$ & $\mathrm{N}^{\prime \prime}$ & $(\%)$ \\
Femea & 15 & $(20,5)$ & 58 & $(79,5)$ & 73 & $(100,0)$ \\
Matcho & 11 & $(22,0)$ & 39 & $(78,0)$ & 50 & $(100,0)$ \\
\hline Total & 26 & $(21,1)$ & 97 & $(78,9)$ & 123 & $(100,0)$
\end{tabular}

Não houve diferença significativa $(P<0,05)$ entre os sexos, em relaçāo ao número de animais positivos.

A média dos comprimentos do eixo maior dos cistos foi de $9,6 \mu \mathrm{m}$, sendo o desvio padrăo de $0,8 \mu \mathrm{m}$ e o coeficiente de variação de $8,3 \%$. A média dos comprimentos do eixo menor dos cistos foi de $6,8 \mu \mathrm{m}$, sendo o desvio padrāo de $0,6 \mathrm{~mm}$ e o coeficiente de variaçăo de $8,8 \%$.

\section{DISCUSSĀO E CONCLUSĀO}

Embora a infecção por Giardia em eqüinos seja considerada rara (Xiao \& Herd, 1994), a ocorrência da giardiase estimada no presente trabalho $(21,1 \%)$ mostra que esta infecçāo em eqüinos é relativamente comum na região estudada.

$O$ resultado encontrado no presente trabalho deve ser considerado como uma subestimativa, em virtude dos eqüinos eliminarem cistos de $G$. duodenalis de forma intermitente (Xiao \& Herd, 1994) e da possibilidade de alguns animais se encontrarem no periodo prepatente da infecção.

A ocorrencia de $G$. duodenalis foi menor em animais com idade menor ou igual a um ano $(18,2 \%)$ do que em animais com idade maior de um ano $(22,8 \%)$, mas esta diferença nāo foi significativa (Tabela 1). Estes resultados concordam com os de Kirkpatrick (1989), que afirmou que a tendēncia dos eqüinos se infectarem ou apresentarem sinais de giardíase, nâo está relacionada com a idade.

Xiao \& Herd (1994) identificaram em eqüinos do sul de Ohio, uma prevalência semelhante entre potros $(29,4 \%)$ e éguas $(27,8 \%)$. Os autores acham que a alta prevalência da giardiase em éguas durante a lacatação pode ser o resultado de um relaxamento imunológico no final da gestaçāo e durante a lactação.

A prevalência da giardiase foi identificada como sendo inversamente proporcional à idade em todos os levantamentos epidemiológicos feitos em outros hospedeiros da região: cẫes de Maringá (Vargas, 1993), gatos de Maringá (Vargas et al. 1994a), ovinos de Cidade Gaúcha (Vargas et al., 1994b) e bovinos de leite do noroeste do Paraná (Rigolon \& Vargas, 1996b).

A ocorrência estimada da infecção por $G$. duodenalis foi semelhante em fêmeas $(20,5 \%)$ e machos $(22,0 \%)$, nāo havendo diferença significativa (Tabela 2).

As dimensóes médias dos cistos de $G$. duodenalis identificados nas fozes dos eqūinos são menores que as registradas por Varela \& Salsamendi (1958), Kulda \& Nohynkova (1977), Bemrick (1968) e Kirkpatrick \& Skand (1985).

Levando em consideração os resultados obtidos nas condiçōes que foi realizado o presente trabalho, podemos concluir que: a ocorrência de $G$. duodenalis em eqüinos do noroeste e norte do Paraná foi de $21,1 \%$; a ocorrência foi maior nos eqüinos com idade menor ou igual a um ano $(18,2 \%)$ do que $\mathrm{cm}$ eqüinos com idade maior do que um ano (22,8\%), mas esta diferença năo foi significativa; a ocorrência foi semelhante entre fêmeas $(20,5 \%)$ e machos $(22,0 \%)$, não havendo diferença significativa e a morfometria mostrou que os cistos de $G$. duodenalis $(9,6 \pm 0,8 \times 6,8 \pm 0,6 \mu \mathrm{m})$ identificados nos eqüinos são menores aos mensurados neste hospedeiro em outros paises e em outros hospedeiros na regiāo estudada. 
VARGAS, L., RIGOLON, L.P. Occurrence of Giardia duodenalis in equines of northwest and north of Paraná states - Brazil. Semina: Ci. Agr., Londrina, v.19, n.1, p.73-75, mar. 1998.

ABSTRACT: 123 stool samples of euine from the northwest and north of Parana State were evaluated for the presence of Giardia duodenalis cysts. The cysts ocurrence was estimated in $21,1 \%$, considereing the variables: age and sex of the animals. The average of cyst size was $9,6 \pm 0,8 \times 6,8 \pm 0,6 \mu \mathrm{m}$. The centrifugal and floating technique acording Faust et al. (1938) with zinc sulfate (specific mass $=1.180 \mathrm{~kg} / \mathrm{m}^{3}$ ) was used. Samples were collected from novembrer 1995 to july 1996.

KEY WORDS: Giardia duodenalis giardiasis, equine, epidemiology.

\section{REFERÉNCIAS BIBLIOGRÁFICAS}

ACHA, P.N., SZYFRES, B. Giardiasis. Zoonosis y enfermedades transmisibles comunes al hombre $y$ a los animales. Washington: Organización Panamericana de la Salud, 1986. p.611-615 (Publicación Cientifica. 503).

BEMRICK. W.J. Giandia in North American horses. Vet. Med. Smal/ Anim Clin. v.63, p.163-165, 1968.

FAUST, E.C., D'ANTONI, J.S, ODOM, V., MILLER, M.J., PEREZ, C., SAWITZ, W., THOMEN, L.F., TOBIE, J., WALKEA, J.H. A critical study of clinical laboratory techniques for the diagnosis of protozoan cysts and helminth eggs in feces. Am. $S$. Trop, Med. Hyg. v.18, p.169-183, 1938.

HEALY, G.R. Giardiasis in perpective: the evidence of animals as a source of human Giardia infections. In: MEYER, E.A. Giardiasis. Amsterdam: Elsevier, 1990. p.305-313.

KIRKPATAICK, C.E. Giardiasis in large animals. Comp. Contin. Educ. Pract. Vet v.11, p.80-84, 1989.

KIFKPATAICK, C.E., SKAND, D.L. Giardiasis in a horse. $U$. An, Vet. Med. Ass. v.187, n.2, p.163-164, 1985.

KULDA, J., NOHYNKOVA, E. Flagellates of the human intestine and of intestines of other species. In: KREIER. J.P. Parasitic Protozoa, New York: Academic Press, 1977. v.2, p.1-138.

MANAHAN, F.F. Diarrhoca in horses with particular reference to a cinronic diarthoea syndrome. Aust. Vot. J. v.46, p.231-234, 1970 .

RIGOLON, L.P., VARGAS, L. Morfometria e cistos por grama de tezes (CPG) de Giardia em bovinos de leite no noroeste do Paråná - Brasil, Rev. UNIMAR. v.18, n.3, p.607-615, 1996a.

RIGOLON, L.P., VARGAS, L. Prevaléncia de giardia $\mathrm{em}$ bovinos do leite no noroeste do Paraná-Brasil. Rev. UNIMAR. v.18, ก.3, p.617-626, $1996 b$.
SCHANTZ, P.M. Parasitic zoonoses in perspective. Int. J. Parasitol. $\checkmark .21$, p. 161-170, 1991 .

VARELA, J.C., SALSAMENDI, R. Primer hallazgo en el pais de infección natural de nuestros eqüinos por Giardia equi Fanthan, 1921. An, Fac, vet. (Uruguay). v.8, p.165-171, 1958.

VARGAS, L. Prevaléncia de giardia em càes de Maringá - Paraná - Brasis, Sāo Paulo : USP, 1993. 101p. Tese (Doutorado em Parasitologia) - Instituto de Cièncias Biomédicas, Universidade de Sào Paulo, 1993.

VARGAS, L.,FERAEIRA, C.S., BOMBO, D.L. Provalència de Gianoía duodenalis om cāes e gatos de Maringá - Paraná - Brasil. Anuario - CCA (Universidade Estadual de Maringá - Centro de ciências Agrárias). p.73-75, 1995/96a.

VARGAS, L., FERREIRA, C.S., CARVALHEIRA, M.S. Morfometria e cistos por grama de fezes (CPG) de Giardia em ovinos de Cidade Gaúcha - Paraná - Brasil. Rev. UNIMAR, v.16, n.2, 0.281-287, 1994a.

VARGAS, L., FERREIRA, C.S., CARVALHEIRA, M.S. Prevalència de giandia em ovinos de Cidade Gaúcha - Paraná - Brasil. Rev. UNIMAR, v.16, n.2, p.289-296, 1994b.

VARGAS, L. RIGOLON, L.P., FERREIRA, C.S. Provalència de Giardia duodenalis em ruminantes do noroeste do Paraná Brasil. Anuario - CCA Runiversidade Estadual de Maringá Centro de Ciencias Agrarias). P.69-71, 1995/96b.

XIAO, L. Giardia infoction in farm animals. Parasitol. Today v.10, n.11, p.436-438. 1994.

XIAO, L., HERD, R.P. Epidemiology of equine Cryptosporidiumand Giardia infections. Equine Vot. J. v.26, n.1, p.14-17, 1994. 